\title{
Efficacy of Cranial Doppler Ultrasound in Diagnosis of Early- Onset Neonatal Sepsis
}

Ahmed Adel Ahmed Elheity ${ }^{1, *}$ MBBCh; Ahmed Mohsen Abdelhakeem ${ }^{1}$ MD; Sameh Abdelaziz Ahmed ${ }^{1}$ MD; Mohammad Abolwafa Ahmad ${ }^{2}$ MD

*Corresponding Author:

Ahmed Adel Ahmed

Elheityahmed.adel.elheity@gmail.com

Received for publication June23, 2021; Accepted July23, 2021; Published onlineJuly23, 2021.

CopyrightThe Authors published by Al-Azhar University, Faculty of Medicine, Cairo, Egypt. Users have the right to read, download, copy, distribute, print, search, or link to the full texts of articles under the following conditions: Creative Commons AttributionShare Alike 4.0 International Public License (CC BY-SA 4.0).

doi:10.21608/aimj.2021.78290.1489

${ }^{1}$ Pediatrics Department,Faculty of Medicine, Al-Azhar University, Cairo , Egypt.

${ }^{2}$ Radiology Department,Faculty of Medicine, Al-Azhar University, Cairo, Egypt.

\begin{abstract}
Background: Neonatal sepsis is considered a major neonatal issue and associated with a high rate of mortality in newborns. Because there is a lack of studies concerning early hemodynamic changes of cerebral circulation in neonatal sepsis, so our research aims to assess the cerebral blood flow (CBF) changes in newborns with early onset neonatal sepsis (EONS) and to clarify the role of cranial Doppler Ultrasound in EONS diagnosis.

Aim of The Work: To evaluate cerebral hemodynamic changes using transcranial Doppler in newborns with EONS.

Patient and Methods: This cross sectional study with 50 neonates involved in the study. The neonates were classified into 2 groups: Case Group (25 neonates) with a diagnosis of early-onset neonatal sepsis and control group (25 neonates) without clinical manifestations of sepsis. Examination of all neonates using Transcranial Doppler (TCD) was performed within seventy-two hours after birth. Measurement of cerebral blood flow velocity (CBFV) in two major cerebral vessels: The anterior cerebral artery (ACA) and middle cerebral artery (MCA) with documentation of doppler data: The peak systolic velocity (PSV), pulsatility index (PI), and resistive index (RI). Data were statistically analyzed followed by calculation of specificity, sensitivity, positive and negative predictive value at selected cut-off values of CBFV parameters. Results: Sepsis group showed statistically significant changes in doppler indices with increased PSV and deceased PI and RI in both arteries.

Conclusion: The present study demonstrates changes in CBFV measured by transcranial Doppler in newborns with EONS which can be used as noninvasive bedside investigation.
\end{abstract}

Keywords: Cerebral; Doppler; Hemodynamics; Neonates; Sepsis.

Disclosure: The authors have no financial interest to declare in relation to the content of this article. The Article Processing Charge was paid for by the authors.

Authorship: All authors have a substantial contribution to the article.

\section{INTRODUCTION}

The third leading cause of neonatal mortality is neonatal sepsis. It constitutes about $13 \%$ of global neonatal deathsand approximately $42 \%$ of first weak deaths in neonates. ${ }^{1}$

Neonatal sepsis defines a systemic disease that occurs due to bacterial, viral, or fungal infection and associated with clinical manifestation and hemodynamic changes. The frequency is between 2 and 22 in every thousand live births globally. ${ }^{2,3}$

Regarding the onset of manifestation, Sepsis can be classified into two groups. When clinical features manifest within the first three days $(<72 \mathrm{~h})$ of life this can be defined as Early-onset neonatal sepsis. while the term late-onset sepsis defines features of sepsis that manifest beyond $72 \mathrm{~h}$ of birth. ${ }^{4}$

The function of cerebral autoregulation is not mature enough in neonates so neonates are more liable to cerebral blood flow fluctuations. It is also recognized that both hypo and hyper-perfusion can cause brain damage in neonates with increased risk of cerebral hemorrhage occurred with hyper-perfusion and increased risk of ischemia occurred with hypo- perfusion. Therefore, studying the neonatal cerebral blood flow and velocities in sepsis is of concern to identify any alteration of cerebral circulation. ${ }^{5}$

There is a lack of literatures that studies the relationship between neonatal sepsis and early changes occurring in cerebral blood flow (CBF). ${ }^{6}$

Early hours assessment of the CBF and brain hemodynamic in newborns by using transcranialdoppler have a major role in the diagnosis of neonatal brain damage and identify the Cause of neonatal morbidity.

The aim of our study is the evaluation of CBF alteration and changes of Doppler indices in neonates with EONS and to clarify the role of cranial Doppler Ultrasound as a diagnostic tool for EONS.

\section{PATIENTS AND METHODS}

Our study was a cross-sectional analytical study conducted over 6 months duration and a total of 50 neonates joined the study.We divide the neonate into 2 groups: (Case group) contained 25 neonates with diagnosis of EONS and (Control group) contained 25 
neonates without any clinical manifestations of sepsis.

The patients were recruited from the NICU, AlAzhar university hospitals (from September 2020 to March 2021), after the approval of the committee of ethics in the faculty of medicine, Al-Azhar University of Cairo.

The parents or caregivers of all neonates gave an oral consent before being involved in the study and after being fully informed about the study and its circumstances.

\section{Inclusion criteria}

The case group included neonates who presented within $72 \mathrm{~h}$ after delivery with either clinical manifestation of sepsis or a positive septic screen and/or positive culture.

Sepsis clinical manifestations include temperature imbalance, poor activity or suckling, feeding intolerance, lethargy, abnormal neonatal reflexes, hypotonia, respiratory distress, frequent or severe apnea, abnormal serum glucose level, or metabolic acidosis.

The positive septic screen includes hematological scores of Rodwell $\geq 3^{8}$ and $\mathrm{C}$ reactive protein (CRP) $>6 \mathrm{mg} / \mathrm{dl}$.

\section{Exclusion criteria}

History of eclampsia in the mother or use of medication during pregnancy like tocolytic or steroids.

Neonates who had diseases like: Multiple congenital anomalies, major congenital heart disease, hemolytic anemia, perinatal asphyxia, inborn error of metabolism, meconium aspiration syndrome, or surgical problems.

Any neonate at the time of Doppler examination had: Abnormal ABG, hemodynamic instability and receive inotropes or vasopressors, severe lung pathology needs mechanical ventilation, receiving drugs like anticonvulsants, caffeine citrate, or theophylline.

\section{Clinical and laboratory assessment:}

Perinatal history in detail was collected.

Full general and local examinations were performed at the time of admission for exclusion of congenital anomalies or any systemic disease included in exclusion criteria.

Basic investigations withdrawn and sent to the lab: Complete blood count, blood film, CRP, ESR, plasma glucose level, serum electrolytes, ABG, coagulation profile, AST, ALT, urea, and creatinine.

According to our NICU protocol, all neonates enrolled in the study received routine neonatal care.

\section{Doppler examination}

Within 72 h after delivery, all neonates undergo Transcranial Doppler examination with assessment of CBFV. All ultrasound examinations were carried out by the same sonographer, using GE Medical
Systems (China) Co., Ltd. DESC: Vivid e. Using sector probe (6S) with a bandwidth of $2.5-7.0 \mathrm{MHz}$. Examination of the anterior cerebral artery was conducted through the anterior fontanelle, while examination of the middle cerebral artery was conducted through the transtemporal approach (Figure 1). The analysis of spectral wave was performed at a $60^{\circ}$-degree angle. Throughout the examination, gain, volume, and power were maintained. After selection of the peak systolic velocity (PSV) and end diastolic velocity (EDV) on the doppler waves, the pulsatility index and resistive index were calculated. (Figure 2).

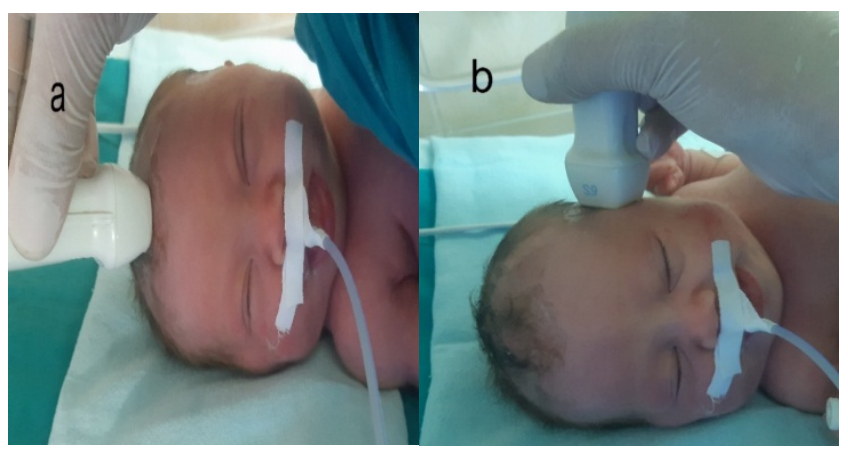

Fig. 1: Doppler examination of cerebral vessels (a) ACA examination through the anterior fontanel (b) MCA examination through temporal view.

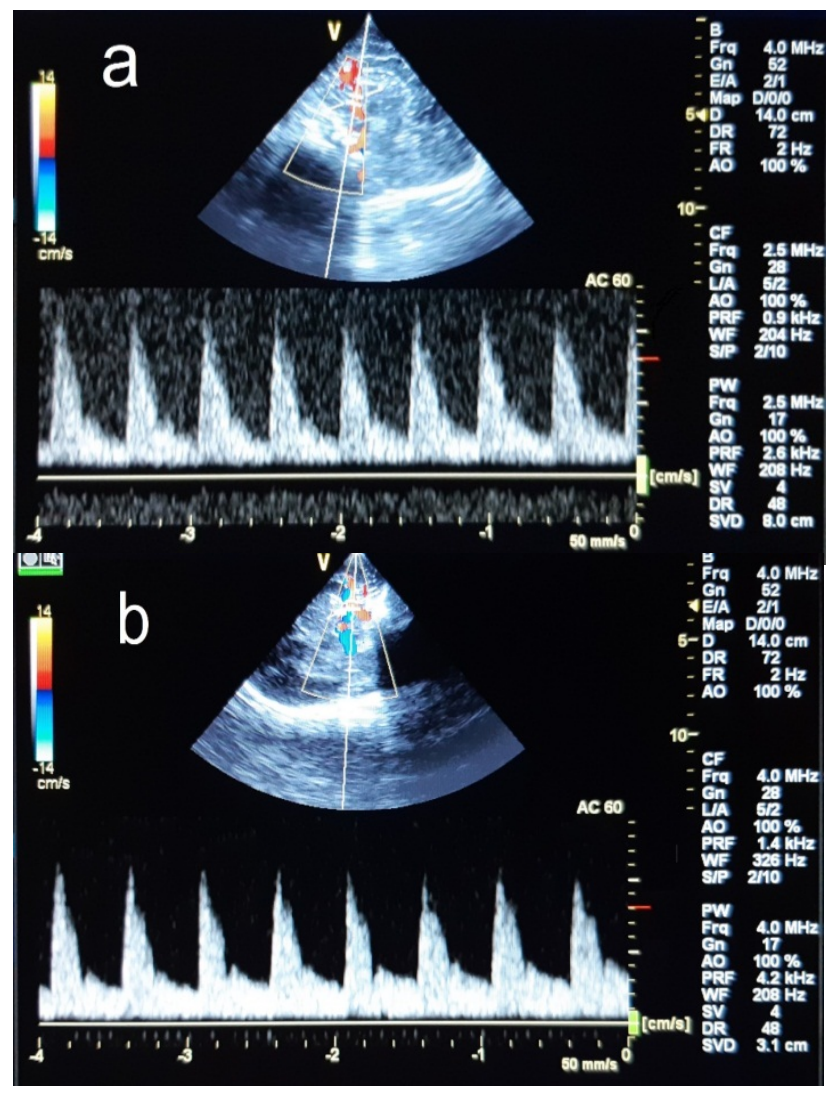

Fig. 2: Doppler waves during examination of (a) ACA (b) MCA.

\section{Statistical analysis}

The statistical application IBM SPSS version 20.0. (Armonk, NY: IBM Corp.) was used to analyze the 
data statistically. The percentages were used to represent the frequencies and proportions of the various variables. The Kolmogorov-Smirnov test was employed to ensure that the distribution was normal. For normally distributed independent samples, the Student t-test was employed to compare numerical variables between study groups, while MannWhitney $U$ test was employed to compare independent samples when not normally distributed. The proportions between groups were compared using the Chi-square test. When the P-value less than 0.05 , it was considered statistically significant.

\section{RESULTS}

\section{Patients}

The study included fifty neonates who were admitted to the NICU following delivery and met the inclusion and exclusion criteria. The sepsis group (case group) included 25 neonates with clinical manifestations and positive septic screen for EONS, while the non-sepsis group (control group) included 25 neonates with negative septic screen and no manifestations of EONS.

Neither group of neonates required resuscitation in the delivery room, and the median value of Apgar score of both groups at 5 minutes was 8 .

Meningitis and encephalitis were ruled out in all neonates based on clinical and laboratory evaluation. Only 7 of sepsis patients had a positive blood culture (28\%). Gram-negative infections account for $71 \%$ (5/7) of culture-positive sepsis. The most commonly isolated organism was E. coli 3 (42\%), followed by Klebsiellapneumoniae (28\%) in 2 cultures and Pseudomonas aeruginosa in one culture (14\%). While only one culture of a gram-positive bacterium (Staphylococcus epidermidis) was identified (14\%).

Twelve of the fifty neonates (24\%) were examined on day one (within $24 \mathrm{~h}$ after delivery), eighteen (36\%) on day two (within 24-48 h after delivery), and twenty (40\%) on day three (within 48-72 h after delivery).

Five neonates without sepsis were examined on day one, ten neonates on day two, and ten neonates on day three. While in neonates with sepsis seven were examined on the first day of life, eight on the second day, and ten on the third day.

Fourteen of the neonates (28\%) were preterm while 36 (72\%) were term. There were 20 term and 5 preterm neonates in the control group, while 9 preterm and 16 term neonates in the sepsis group.

There were 18 (36\%) females and 32 (64\%) males out of a total 50 neonates. There were $17(68 \%)$ males and 8 (32\%) females among neonates who had sepsis. While in neonate without sepsis there were 15 (60\%) male and 10 (40\%) females. Male were more common to develop sepsis in neonates.

The number of neonates who were delivered vaginally was 16 (32\%) whereas the number of neonates who were delivered via cesarean section was 34 (68\%). There were 19 (76\%) cesarean sections and 6 (24\%) vaginal deliveries among neonates with sepsis. While in neonates without sepsis there were $15(60 \%)$ cesarean section and 10 (40\%) vaginal delivery. So, the results show that sepsis is more common in neonates who were delivered via cesarean section.

In our study statistics show that there was a significant decrease in birth weight in the sepsis group (Mean=2.174 kg), as compared with the control group $($ Mean=2.578 kg) $($ Table 1$)$.

\begin{tabular}{|c|c|c|c|c|c|}
\hline & \multicolumn{2}{|c|}{$\begin{array}{l}\text { Sepsis } \\
(n=25)\end{array}$} & \multicolumn{2}{|c|}{$\begin{array}{l}\text { Control } \\
(\mathrm{n}=25)\end{array}$} & \multirow[b]{2}{*}{ p-Value } \\
\hline & No. & $\%$ & No & $\%$ & \\
\hline Male & 17 & $68 \%$ & 15 & $60 \%$ & \multirow{2}{*}{0.55} \\
\hline Female & 8 & $32 \%$ & 10 & $40 \%$ & \\
\hline Full-term & 16 & $64 \%$ & 20 & $80 \%$ & \multirow{2}{*}{0.20} \\
\hline Pre-term & 9 & $36 \%$ & 5 & $20 \%$ & \\
\hline CS & 19 & $76 \%$ & 15 & $60 \%$ & \multirow{2}{*}{0.22} \\
\hline VD & 6 & $24 \%$ & 10 & $40 \%$ & \\
\hline $\begin{array}{l}\text { Gestational } \\
\text { Age (Weeks) }\end{array}$ & \multicolumn{2}{|c|}{$\begin{array}{c}36.9 \pm 1.5 \\
(34-39)\end{array}$} & \multicolumn{2}{|c|}{$\begin{array}{c}37.2 \pm 1.5 \\
(34-39)\end{array}$} & 0.41 \\
\hline $\begin{array}{l}\text { Birth-weight } \\
\text { (grams) }\end{array}$ & \multicolumn{2}{|c|}{$\begin{array}{l}2174.8 \pm 679 \\
(1550-3700)\end{array}$} & \multicolumn{2}{|c|}{$\begin{array}{l}2578.4 \pm 669 \\
(1690-3800)\end{array}$} & 0.039 \\
\hline $\begin{array}{c}\text { Days of } \\
\text { NICU } \\
\text { admission }\end{array}$ & \multicolumn{2}{|c|}{$\begin{array}{l}22.96 \pm 11.2 \\
\quad(7-42)\end{array}$} & \multicolumn{2}{|c|}{$\begin{array}{c}10 \pm 8.88 \\
(2-32)\end{array}$} & 0.0001 \\
\hline
\end{tabular}

Table 1: Demographic data difference between case and control groups: there was a considerable difference among the studied groups regarding birthweight and days of stay in NICU.

\section{Data of Doppler examination}

The results of the anterior and middle cerebral arteries examination demonstrate a statistically significant difference in doppler indices between the case and control groups: Septic group had a high PSV but low RI and PI. (Table 2). 


\begin{tabular}{|c|c|c|c|c|c|c|}
\hline \multirow{2}{*}{ Doppler data } & \multicolumn{2}{|c|}{$\begin{array}{c}\text { Sepsis } \\
(\mathrm{n}=25)\end{array}$} & \multicolumn{2}{c|}{$\begin{array}{c}\text { Control } \\
(\mathrm{n}=25)\end{array}$} & \multirow{2}{*}{$\begin{array}{c}\text { p- } \\
\text { Value }\end{array}$} \\
\cline { 2 - 7 } & $\begin{array}{c}\text { Mean } \pm \\
\text { SD }\end{array}$ & Median & $\begin{array}{c}\text { Mean } \pm \\
\text { SD }\end{array}$ & Median & \\
\hline \multirow{5}{*}{ ACA } & $\begin{array}{c}\text { PS } \\
\text { V }\end{array}$ & $65.5 \pm 8.8$ & 67.9 & $42.2 \pm 10.9$ & 38.6 & 0.0001 \\
\cline { 2 - 7 } & PI & $1.01 \pm 0.17$ & 1.01 & $1.42 \pm 0.13$ & 1.42 & 0.0001 \\
\cline { 2 - 7 } & RI & $0.57 \pm 0.07$ & 0.58 & $0.73 \pm 0.04$ & 0.76 & 0.0001 \\
\hline \multirow{4}{*}{ MCA } & $\begin{array}{c}\text { PS } \\
\text { V }\end{array}$ & $67.8 \pm 7$ & 68.6 & $43.4 \pm 9$ & 39.9 & 0.0001 \\
\cline { 2 - 7 } & PI & $0.94 \pm 0.17$ & 0.9 & $1.38 \pm 0.18$ & 1.38 & 0.0001 \\
\cline { 2 - 7 } & RI & $0.55 \pm 0.08$ & 0.52 & $0.72 \pm 0.03$ & 0.73 & 0.0001 \\
\hline
\end{tabular}

ACA: anterior cerebral artery; MCA: middle cerebral artery; PSV: peak systolic velocity; PI: pulsatility index; RI, resistivity index; p-value: Probability value

Table 2: Comparison of Doppler data between neonates without sepsis and neonates with sepsis measured in ACA and MCA: This table shows that there was statistically significant increased PSV with decreased PI and RI in both ACA and MCA in the septic group.

The diagnostic value of Doppler indices measured in the ACA, and MCA show that the sensitivity of the PI of the ACA and MCA was 96\%. While the specificity of RI of the MCA was $96 \%$ (Table 3).

\begin{tabular}{|c|c|c|c|c|c|c|}
\hline $\begin{array}{c}\text { Doppler } \\
\text { data }\end{array}$ & $\begin{array}{c}\text { AU } \\
\text { C }\end{array}$ & $\begin{array}{c}\text { Cut- } \\
\text { off }\end{array}$ & Sensitivity & Specificity & + LR & -LR \\
\hline ACA_PSV & 0.896 & $\geq 49.3$ & 88.00 & 92.00 & 11.00 & 0.13 \\
\hline ACA_PI & 0.973 & $\leq 1.24$ & 96.00 & 88.00 & 8.00 & 0.045 \\
\hline ACA_RI & 0.959 & $\leq 0.66$ & 92.00 & 92.00 & 11.50 & 0.087 \\
\hline MCA_PSV & 0.96 & $\geq 55.4$ & 92.00 & 92.00 & 11.50 & 0.087 \\
\hline MCA_PI & 0.952 & $\leq 1.22$ & 96.00 & 80.00 & 4.80 & 0.050 \\
\hline MCA_RI & 0.919 & $\leq 0.64$ & 88.00 & 96.00 & 22.50 & 0.13 \\
\hline AUC: AI & & & & & & \\
\hline
\end{tabular}

AUC: Area under curve; p-value: Probability value; CI: Confidence Intervals; +LR: Positive likelihood ratio; -LR: Negative likelihood ratio.

Table 3: Doppler Parameters in the anterior and middle cerebral arteries at selected cut-off point and their diagnostic value.

Outcome:

During the hospital stay, 4 neonates (16\%) from the sepsis group develop intraventricular hemorrhage (IVH).
There was a significant increase in days of NICU admission among the sepsis group (Mean=22.9days) as compared with the control group (Mean=10days).

There was also an increased mortality rate in the sepsis group 8 (32\%) as compared with neonates without sepsis $2(8 \%)$.

\section{DISCUSSION}

Neonatal sepsis is considered a systemic disease caused by an infection that affects newborns during the first 28 days after birth and leads to many morbidity and mortality in neonates. ${ }^{9}$

The inflammatory process of neonatal sepsis activates the immune system of the fetus, causing fetal inflammatory response syndrome (FIRS). Initially, FIRS is asymptomatic, but the inflammatory process might progress to systemic inflammatory response syndrome (SIRS), which is clinically identified as EONS after birth. ${ }^{5}$

There are many factors such as impaired cerebral autoregulation, disruption to the blood-brain barrier (BBB), oxidative stress, and direct inflammatory effects of free radicals and cytokines on glial cells, may contribute to the pathophysiology of sepsisinduced encephalopathy. ${ }^{10}$

The mechanism by which these cytokines reach the brain to make damage is still uncertain. Cytokines cross BBB causing its damage and lead to increased permeability. Microglia and astrocytes are also able to produce proinflammatory cytokines during inflammation. These proinflammatory cytokines may cause excitotoxicity by changing glutamate reuptake, triggering the release of free radicals, or inducing the local synthesis of nitric oxide which causes vasodilatation. ${ }^{5}$

Rapid changes in cerebral blood flow may lead to acute and chronic neonatal morbidity. So CBFV changes play a vital role in neonatal brain injury. ${ }^{11}$

Children with neonatal sepsis are more likely to develop cognitive and neuromotor difficulties around the age of 12 months, according to some researches. ${ }^{12}$

Data of Doppler examination in our study reveal considerably low (RI and PI) with high PSV in ACA \& MCA have been documented in sepsis, reflecting a generalized increase in $\mathrm{CBF}$ and cerebral vasodilatation as an early response to sepsis, as examination were done within the first 3 days after birth in all neonates.

Our results were in accordance with those of Sriparna et al. ${ }^{6}$ who found a significant decrease in (RI and PI) with an increase in PSV in neonates with EONS during the examination of (Vertebral, middle cerebral, and internal carotid arteries) within the first 24 hours after birth, and Koch et al. ${ }^{13}$ who proved that full terms neonates with maternal chorioamnionitis exhibited lower resistance in most cerebral arteries than neonates with no maternal history of chorioamnionitis.

The peak systolic velocity values in our study showed higher Mean values than that recorded by 
Sriparnaet al. ${ }^{6}$, which can be explained by the difference in timing of transcranial Doppler examination, and according to researches CBFV rise progressively with the postnatal age. ${ }^{14}$

Also, our results were in accordance with those of Hashem et al. ${ }^{5}$ who found increased peak systolic velocity and lower resistance in neonates with EONS by examination of the ACA and MCA in preterm neonates at the third day after birth, and El Shimy et al. ${ }^{15}$ who found that neonates with EONS had raised cerebral blood flow and neuron-specific enolase in cord blood.

The sensitivity of the pulsatility index in our study was the highest of all measures in ACA and MCA with a sensitivity of $96 \%$.

In the different studies, there were some variations in the measurements of ACA and MCA parameters in neonates with and without sepsis. These variances can be due to the Doppler measurements being performed at different times and the number of culture-positive patients being different in each study.

The role of transcranial Doppler in the assessment of EONS is significant, and it can be used as a bedside test for early diagnosis of SIRS and EONS. It has been recognized that there is an increase in the CBF even before the appearance of sepsis manifestations, implying that the inflammatory process first affects the cerebral circulation before it can affect any other body system.

Our study has been supported by strict following of inclusion and exclusion criteria. As we ruled out any neonate with perinatal asphyxia, hydrodynamic instability, needed mechanical ventilation, showed severe ABG alteration, and those were receiving anticonvulsants or caffeine at the time of TCD assessment because these conditions could alter the CBFV measurements.

Because our study was cross-sectional, so we assessed the CBFV one-time and we couldn't evaluate the long-term changes in CBFV or compare it with the adverse effect, some studies have found that the risk of neuromotor and cognitive developmental problems at age of one-year- old was three times more in children who previously had neonatal sepsis. ${ }^{13}$

Limitations of the study: Because our study was cross-sectional with a one-time evaluation of CBFV, we were unable to evaluate changes in CBFV over time and compare them with the unfavorable effect.

\section{CONCLUSION}

The current study found that neonates with EONS have altered cerebral hemodynamics with increased $\mathrm{CBF}$ and decrease resistance.

In our study, we document the cut-off value at which best sensitivity and specificity of EONS diagnosis. CBF assessment by Doppler ultrasound in the first few hours after birth might be accepted as a bedside investigation, noninvasive with good diagnostic and prognostic value. It can be thought of as a complement to the laboratory investigations which are invasive and require a large amount of blood samples.

Other advantages of Doppler examination rather than noninvasiveness, include portability, accuracy, lack of ionizing radiation, cost-effectiveness, and the ability to examine cranial anatomy and evaluate CBFV .

The greatest benefit from Doppler examination can be obtained when the examination repeated serially and performed with the initial establishment of treatment.

\section{REFERENCES}

1. Zea-Vera A, Ochoa TJ. Challenges in the diagnosis and management of neonatal sepsis. $J$ Trop Pediatr. 2015;61(1):1-13.

2. Dong Y, Glaser K, Speer CP. Late-onset sepsis caused by Gram-negative bacteria in very low birth weight infants: a systematic review. Expert Rev Anti Infect Ther. 2019;17(3):177-88.

3. Shane AL, Sánchez PJ, Stoll BJ. Neonatal sepsis. Lancet. 2017;390(10104):1770-80.

4. Puopolo KM, Benitz WE, Zaoutis TE; COMMITTEE ON FETUS AND NEWBORN; COMMITTEE ON INFECTIOUS DISEASES. Management of Neonates Born at $\geq 35$ 0/7 Weeks' Gestation with Suspected or Proven Early-Onset Bacterial Sepsis. Pediatrics. 2018;142(6): e20182894.

5. Hashem RH, Abdalla YE, Mansi YA, Shaaban MH, El Kiki HA. Transcranial Doppler evaluation of cerebral hemodynamic alteration in preterms with early onset neonatal sepsis. Artery Research. 2017; 19:83-90.

6. Basu S, Dewangan S, Shukla RC, Anupurva S, Kumar A. Cerebral blood flow velocity in earlyonset neonatal sepsis and its clinical significance. Eur J Pediatr. 2012;171(6):901-9.

7. Molnár L, Fülesdi B, Németh N, Molnár C. Sepsis-associated encephalopathy: A review of literature. Neurol India. 2018;66(2):352-61.

8. Rodwell RL, Leslie AL, Tudehope DI. Early diagnosis of neonatal sepsis using a hematologic scoring system. J Pediatr. 1988;112(5):761-7.

9. Tewabe T, Mohammed S, Tilahun Y, Melaku B, Fenta M, Dagnaw T, Belachew A, Molla A, Belete $\mathrm{H}$. Clinical outcome and risk factors of neonatal sepsis among neonates in FelegeHiwot referral Hospital, Bahir Dar, Amhara Regional State, North West Ethiopia 2016: a retrospective chart review. BMC Res Notes. 2017;10(1):265.

10. Chaudhry MT, Ling TC, Hussain SA, Manzoor A. Minimizing thermal stress for data center servers through thermal-aware relocation. ScientificWorldJournal. 2014; 2014:684501.

11. Rhee CJ, da Costa CS, Austin T, Brady KM, Czosnyka M, Lee JK. Neonatal cerebrovascular autoregulation. Pediatr Res. 2018;84(5):602-10. 
12. Ratnaparkhi CR, Bayaskar MV, Dhok AP, Bhende V. Utility of Doppler ultrasound in early-onset neonatal sepsis. Indian $J$ Radiol Imaging. 2020;30(1):52-8.

13. Koch FR, Wagner CL, Jenkins DD, Caplan MJ, Perkel JK, Rollins LG, Katikaneni LD, Mulvihill DM. Sex differences in cerebral blood flow following chorioamnionitis in healthy term infants. J Perinatol. 2014;34(3):197-202.

14. Vasiljević B, Gojnić M, Maglajlić-Djukić S, Antonović O. [Normal values of cerebral blood flow velocities in neonates]. SrpArhCelokLek. 2010;138(3-4):186-91.

15. El Shimy MS, El-Raggal NM, El-Farrash RA, Shaaban HA, Mohamed HE, Barakat NM, Farag AS, El Zohiery AK, Shaaban MAA, Salama DH. Cerebral blood flow and serum neuron-specific enolase in early-onset neonatal sepsis. Pediatr Res. 2018;84(2):261-6. 